\title{
Focused Ion Beam Surface Preparation for Plasma Facing Materials
}

\section{Daniel Morrall and Chad Parish}

\section{Oak Ridge National Lab, Oak Ridge, Tennessee, United States}

Focused ion beam surface preparation for plasma facing materials Tungsten is one of the most important nuclear fusion structural materials for use as a plasma facing component in fusion reactors. However, in a critical range of temperatures a unique surface morphology known as "nanofuzz" forms when exposed to He-containing plasma. The so-called nanofuzz grows with increased exposure times, yet the tendrils remain in the size range of $10 \mathrm{~s}$ of nanometers of diameter, forming a many-microns thick layer [1]. Understanding the exact formation mechanism of nanofuzz requires identifying structures and surface morphology within the top 10 nanometers of a tungsten sample after exposure to various He plasma and temperature conditions.

Advanced microstructure characterization of nanofuzz can be completed through transmission electron microscopy (TEM) on properly prepared samples. Of the various TEM sample preparation techniques, focused ion beam (FIB) is the most appropriate for observing near-surface $(<5 \mu \mathrm{m})$ sample behavior. However, the FIB process is inherently damaging to a sample, particularly at the surface. Typically, the ion source for a FIB equipped scanning electron microscope (SEM) is gallium, which is used to sputter sample material and in tandem with another source (i.e. Pt, C, or W) for creating deposited layers used to protect a sample during the milling process. Deposition of the protective layer involves using the incident $\mathrm{keV}$-range ion beam to drive a reaction in the source gas molecules adsorbed to the surface. Even at low energies, implantation can occur in the sample to a depth of $10 \mathrm{~s}$ of $\mathrm{nm}$. Both the $\mathrm{Ga}+$ ions and the deposition material (e.g., Pt) mixes with the sample surface, thereby ruining shallow regions of interest.

Preventing surface mixing of ion and deposition material with parent material is the focus of this presentation. Using permanent marker deposition (PMD) to protect a sample surface with an amorphous carbon layer has been done in the past with some success, however directly applying a marker to a material surface may be damaging to fragile surface structures [2]. In the current work we attempted to determine a more universal means of sample surface protection that could be applied to even highly radioactive materials. We used a combination of electron beam deposition with a carbon-based deposition material in a layered structure with Pt (Fig. 1) followed by a low energy (16kV) Ga-induced Pt deposition to create samples without any surface intermixing. Energy dispersive $\mathrm{x}$-ray spectography (EDS) analysis comparisons with a sample prepared using only the Ga ion beam and Pt deposition material showed a clear improvement of the surface conditions of the specimen, as indicated by the presence or lack of an intermixed layer of PT/Ga with the parent material near the surface. However, there were some cases when the electron beam deposition of the $\mathrm{C}$ layer was insufficient to prevent $\mathrm{Pt}$ mixing with the $\mathrm{W}$ surface even using the electron beam for Pt deposition, so great care must be taken to ensure that the $\mathrm{C}$ layer is sufficiently thick (Fig. 2) to prevent the permeation of Pt atoms into the W surface.

Proper sample preparation techniques maximize the capabilities of TEM analysis. Using this method has allowed the measurement of very fine He bubbles/cavities in a very shallow depth near the surface, less than $10 \mathrm{~nm}$, of both single and polycrystalline tungsten samples after being exposed to He plasma (see Fig. 2). Additionally, a more in-depth understanding of the formation of an oxide layer after exposure to air was achieved. This analysis has led to new insights into the formation mechanisms of tungsten nanotendrils. [4] 


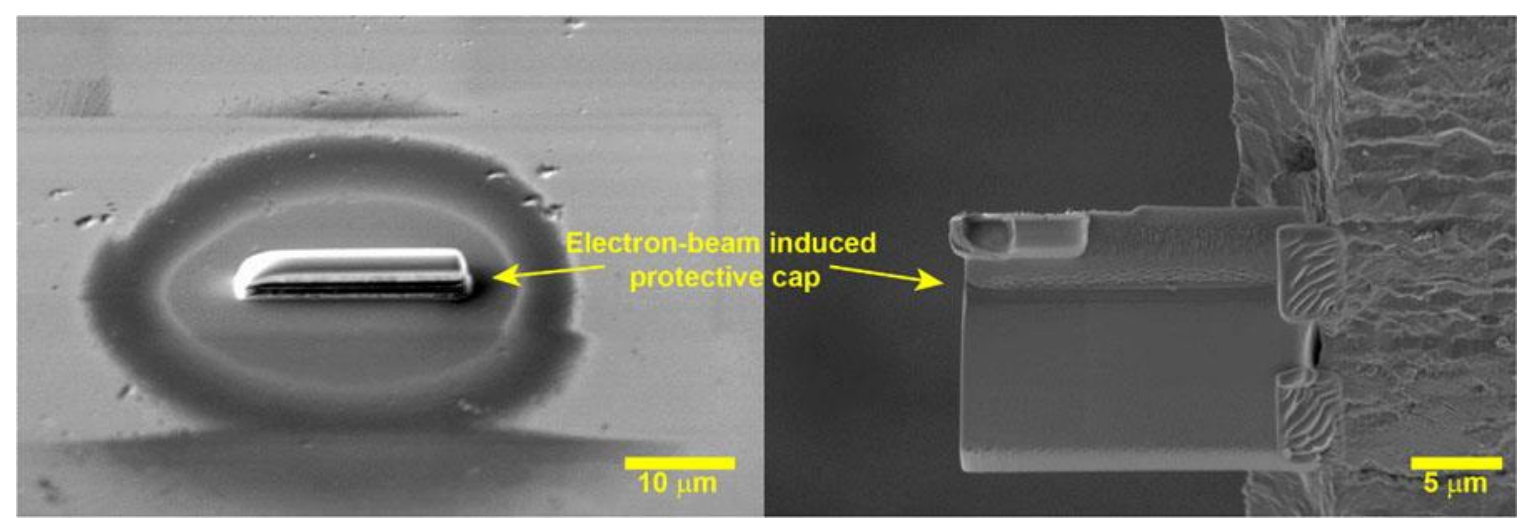

Figure 1. SEM images of tungsten specimen with deposited layers of $\mathrm{C}$ and $\mathrm{Pt}$ using both the electron beam and the ion beam.
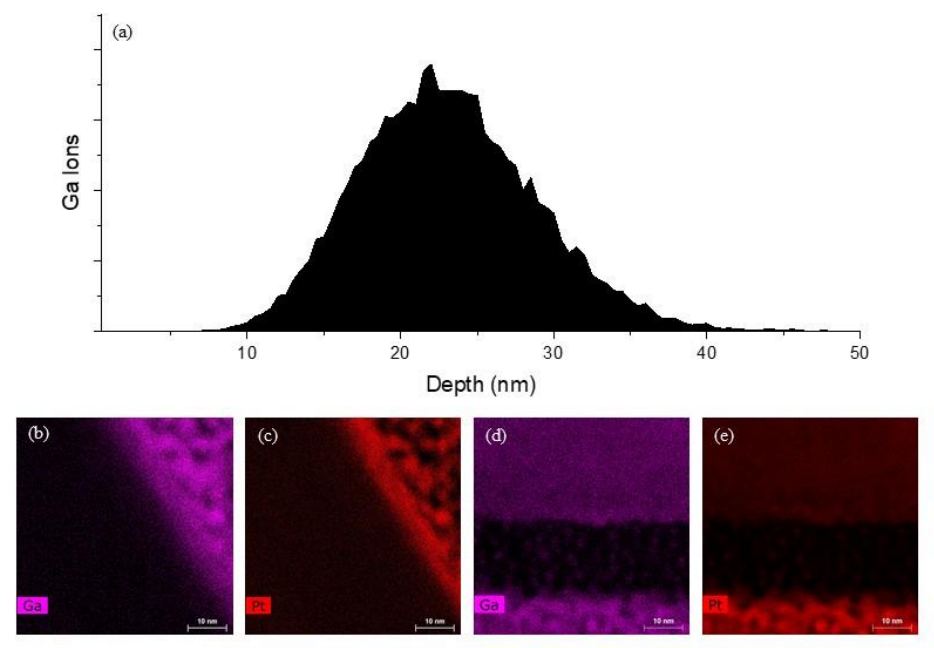

Figure 2. SRIM calculation (a) of $30 \mathrm{keV} \mathrm{Ga}$ ion penetration depth in amorphous carbon (2.3 g/cm3) with EDS analysis and comparison of insufficiently thick $\mathrm{C}$ deposition layer (b\&c) to fully protected surface (d\&e) [3].

\section{References}

[1] C. Parish, K.Wang, R. Doerner, M. Baldwin. Grain orientations and grain boundaries in tungsten nanotendril fuzz grown under divertor-like conditions. Scripta Mat. 127 (2017) 132-135

[2]Y.C. Park, B.C. Park, S. Romankov, K.J. Park, J.H. Yoo, Y.B. Lee, J.M. Yang. Use of permanent marker to deposit a protection layer against FIB damage in TEM specimen preparation. Journal of Microscopy. 255 (2014) 180-187

[3] J.F. Ziegler, J.P. Biersack, M.D. Ziegler. The stopping and range of ions in matter. Lulu press. Ver. 7 (2008)

[4] Research supported by US Department of Energy, Office of Science, Fusion Energy Sciences (including an Early Career Award), under contract number DE-AC05-00OR22725. RPD and MJW supported by DOE grant number: DE-FG02-07ER54912 